Revista Brasileira de Agricultura Irrigada v.11, $\mathrm{n}^{\circ} .8$, p. 2183 - 2193, 2017

ISSN 1982-7679 (On-line)

Fortaleza, CE, INOVAGRI - http://www.inovagri.org.br

DOI: $10.7127 /$ rbai.v11n800707

Protocolo 707.17 - 27/06/2017 Aprovado em 03/08/2017

\title{
COMPARAÇÃO ENTRE MÉTODOS DE ESTIMATIVA DA EVAPOTRANSPIRAÇÃO DE REFERÊNCIA EM PERÍMETRO IRRIGADO NO BAIXO SÃO FRANCISCO
}

\author{
Allan Cunha Barros ${ }^{1}$, Antenor de Oliveira Aguiar Netto ${ }^{2}$, Pedro Robinson Fernandes de
} Medeiros $^{3}$, Thassio Monteiro Menezes da Silva ${ }^{4}$, Kenia Almeida Diniz Albuquerque ${ }^{1}$

\begin{abstract}
RESUMO
A determinação da demanda hídrica de uma cultura é ferramenta importante no manejo da irrigação, em especial em perímetros irrigados como o Jacaré-Curituba, localizado no Baixo São Francisco. A FAO determina que o modelo padrão de estimativa da Evapotranspiração de Referência seja o modelo de Penman-Monteith, no entanto, nem sempre se dispõe de todas as variáveis climatológicas necessárias, devendo então analisar qual deles podem ser utilizados e verificar sua precisão nas estimativas. Assim, o objetivo do trabalho foi comparar diferentes métodos de estimativa da evapotranspiração de referência com o método padrão de PenmanMonteith para o perímetro irrigado Jacaré-Curituba, na cidade de Canindé-SE. Utilizou-se uma séria de dados de 2006 a 2013 e os tratamentos foram baseados na estimativa da ETo pelos modelos de Hargreaves e Samani (HS), FAO - Radiação (FAO-Rad), Blaney-Criddle (BC) e Priestley e Taylor (PT). Utilizou-se parâmetros estatísticos para avaliar a qualidade das estimativas. De todos os modelos avaliados o de HS apresentou os melhores resultados demonstrando seu potencial para o uso na região de estudo.
\end{abstract}

Palavras-chave: Penman-Monteith, Temperatura do ar, Hagreaves e Samani, Jacaré-Curituba

\section{COMPARISON BETWEEN REFERENCE EVAPOTRANSPIRATION ESTIMATION METHODS IN IRRIGATED PERIMETER IN LOW SAN FRANCISCO}

\footnotetext{
ABSTRACT

The determination of the water demand of a crop is an important tool in irrigation management, especially in irrigated perimeters, such as Jacaré-Curituba, located in lower São Francisco. The

${ }^{1}$ Professor(a) do Curso de Agronomia da UFAL, Campus Arapiraca, Av. Manoel Severino Barbosa, Bom Sucesso, Arapiraca, AL, Brasil. email: allan.cunha.barros@gmail.com, keniadiniz@hotmail.com

${ }^{2}$ Professor do Curso de Agronomia da UFS, Av. Marechal Rondon, S/n - Jardim Rosa Elze, São Cristóvão, SE, Brasil. e-mail: antenor.ufs@gmail.com

${ }^{3}$ Professor do Curso de Engenharia Agrícola da UNIVASF, Av. Antônio Carlos Magalhães, 510, Santo Antônio, Juazeiro. e-mail: pedro.fernandes@univasf.edu.br

4 Acadêmico de Agronomia da UFS, Av. Marechal Rondon, S/n - Jardim Rosa Elze, São Cristóvão, SE, Brasil. e-mail:

thassio.ufs@gmail.com
} 
FAO determines that the standard reference evapotranspiration estimation model is the Penman-Monteith model, however, it is not always possible to have all the necessary climatological variables, and must then analyze which can be used and verify their accuracy in the estimates. Thus, the objective of this work was to compare different methods of estimation of reference evapotranspiration with the standard Penman-Monteith method for the JacaréCurituba irrigated perimeter in the city of Canindé-SE. A series of data from 2006 to 2013 was used and the treatments were based on the estimation of ETo by the Hargreaves and Samani (HS), FAO-Radiation (FAO-Rad), Blaney-Criddle (BC) and Priestley and Taylor PT). Statistical parameters were used to evaluate the quality of the estimates. Of all the models evaluated, the HS presented the best results demonstrating its potency for use in the study region.

Keywords: Penman-Monteith, Air temperature, Hagreaves e Samani, Jacaré-Curituba

\section{INTRODUÇÃO}

Os perímetros irrigados no Nordeste exercem papel importante no desenvolvimento econômico com a geração de divisas, abastecimento interno de alimentos, geração de emprego e renda, propiciando melhorias na qualidade de vida da população. Em Sergipe, o Perímetro Irrigado Jacaré Curituba, localiza-se a noroeste do estado, na região do baixo São Francisco, ocupando uma área irrigável de 3.105 ha entre os municípios de Canindé de São Francisco e Poço Redondo (CODEVASF, 2011), possuindo sistemas de irrigação por microaspersão e gotejamento que começaram a ser implantados no ano de 2002.

Em áreas irrigadas, o conhecimento dos dados de evapotranspiração é uma das principais formas de evitar o desperdício de água no momento que for irrigar. O estudo da evapotranspiração é usado para conclusão de projetos e manejos de irrigação, simulação de culturas, planejamento e gerenciamento dos recursos hídricos (LIMA, 2005). Para ter um manejo de irrigação eficiente e racional, devese determinar o consumo de água de cada cultura, mais precisamente em cada fase de seu desenvolvimento.

A metodologia de estimativa da evapotranspiração, via modelo PenmanMonteith (ALLEN et al., 1998), possui a capacidade de ser utilizada em diversas regiões e em diversas escalas temporais, para isso, segundo Ravazzani et al., (2012), são necessários uma gama de dados, dentre eles: temperatura, radiação solar, umidade reativa, e velocidade do vento. Essa grande quantidade de dados torna o modelo PM muitas vezes pouco aplicável em regiões nas quais esse tipo de informação é incipiente, e onde se verifica deficiência na coleta de todas variáveis meteorológicos envolvidas na determinação da Eto (CAMARGO e CAMARGO, 2000).

Segundo Fernandes et al. (2011) o manejo da água e a produtividade das culturas podem ser melhorados em função da confiabilidade dos métodos utilizados para estimar a evapotranspiração das culturas. Segundo Mendonça et al. (2003), vários pesquisadores pelo mundo propuseram modelos indiretos para a estimativa da ETo, com as mais diferentes metodologias e números de variáveis envolvidas. Para escolha do método é necessário saber quais as variáveis meteorológicas disponíveis, a partir daí, verificar quais podem ser aplicados, de acordo com as disponibilidades dessas variáveis climáticas.

Assim, o presente trabalho tem como objetivo comparar diferentes métodos de estimativa da evapotranspiração de referência com o método padrão de Penman-Monteith, para o perímetro irrigado Jacaré-Curituba, na cidade de Canindé-Se, no baixo São Francisco.

\section{MATERIAL E MÉTODO}

O presente trabalho foi feito na Universidade Federal de Alagoas, em parceria 
com a Universidade Federal de Sergipe. O Perímetro Irrigado Jacaré Curituba está localizado no baixo São Francisco, cujas coordenadas geográficas são $9^{\circ} 42^{\prime} \mathrm{S}$ de latitude e $37^{\circ} 44^{\prime} \mathrm{W}$ de Longitude (Figura 1). Segundo classificação Köppen, a região possui clima do tipo Bssh, clima muito quente, semiárido, com 7 a 8 meses seco. Os tipos de solos predominantes são Luvissolos, Planossolos, Vertissolos e Neossolos (CODEVASF, 2011).

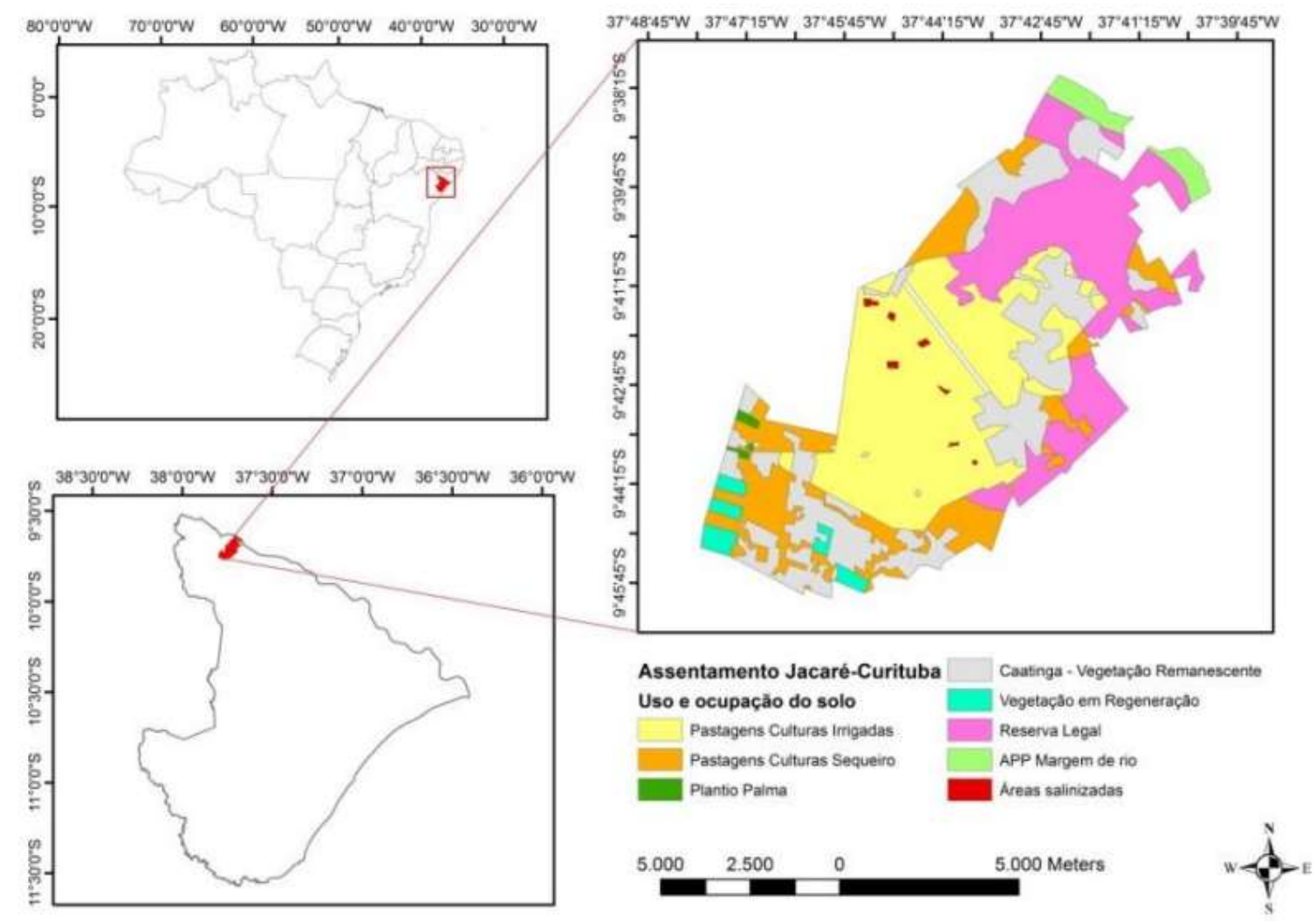

Figura 1. Localização do Perímetro Jacaré-Curituba (Fonte: MENESES et al. 2015)

Os dados relativos às variáveis meteorológicas como temperaturas máxima, mínima e média, umidade relativa do ar, velocidade do vento e radiação solar incidente, foram obtidos através do sítio: www.globalweather.tamu.edu do The National Centers for Environmental Prediction (NCEP). Uma série de dados foi obtida dos anos de 2006 a 2013, registrados em planilha eletrônica.

Utilizaram-se as equações de PenmanMonteith (PM) em comparação com os métodos de Hargreaves e Samani (HS), FaoRadiação (FAO-Rad), Blaney e Criddle (BC) e Priestley e Taylor (PT), para a estimativa da ETo em escala diária.
O método FAO Penman-Monteith é sintetizado na equação 1 (ALLEN et al., 1998).

$E T_{0}=\frac{0,408 \Delta\left(\mathrm{R}_{\mathrm{n}}-\mathrm{G}\right)+\gamma\left(\frac{900 \mathrm{U}_{2}}{T+273}\right)\left(e_{s}-e_{a}\right)}{\Delta+\gamma\left(1+0,34 U_{2}\right)}$

em que:

ETo - Evapotranspiração de referência, mm.dia- ${ }^{1}$;

$\mathrm{Rn}$ - radiação líquida, $\mathrm{MJ} \mathrm{m}^{-2} \mathrm{~d}^{-1}$;

$\mathrm{G}$ - fluxo de calor no solo, $\mathrm{MJ} \mathrm{m}^{-2} \mathrm{~d}^{-1}$;

$\mathrm{T}$ - temperatura média do ar, ${ }^{\circ} \mathrm{C}$;

$\mathrm{V}$ - velocidade média do vento a $2 \mathrm{~m}$ de altura, $\mathrm{m} \mathrm{s}^{-1}$;

$\left(\mathrm{e}_{\mathrm{s}}-\mathrm{e}_{\mathrm{a}}\right)$ - déficit de pressão de vapor, $\mathrm{kPa}$;

$\Delta$ - curva de pressão de vapor, $\mathrm{kPa}^{\circ} \mathrm{C}^{-1}$; 
$\gamma$ - constante psicrométrica, $\mathrm{kPa}^{\circ} \mathrm{C}^{-1}$, e 900 fator de conversão.

$\mathrm{O}$ método descrito em Hargreaves e Samani (1985), foi desenvolvido na Califórnia, em condições semiáridas, a partir de dados obtidos em lisímetros com gramado e descrito conforme equação 2 (PEREIRA et al., 1997).

$$
\text { ETo }=a \times \frac{R a}{2,45} \times\left(T_{\max }-T_{\min }\right)^{b} \times\left(T_{\text {média }}+c\right)
$$

em que:

ETo - Evapotranspiração de referência, mm.dia- ${ }^{1}$;

$\mathrm{R}_{\mathrm{A}}$ - radiação no topo da atmosfera, $\mathrm{MJ} \cdot \mathrm{m}^{-2} \cdot \mathrm{s}^{-}$ 1 ;

$\mathrm{T}_{\max }$ - temperaturas máxima do ar, ${ }^{\circ} \mathrm{C}$;

$\mathrm{T}_{\min }$ - temperaturas mínima do $\mathrm{ar},{ }^{\circ} \mathrm{C}$;

$\mathrm{T}_{\text {média }}$ - temperaturas média do ar, ${ }^{\circ} \mathrm{C}$;

$a, b$ e c - coeficientes da equação.

O método FAO-Radiação é descrito por Doorenbos e Pruitt (1975), demandando observações de insolação, nebulosidade ou radiação, além da temperatura do ar. Pode ser empregado na ausência de dados de umidade relativa e velocidade do vento requerendo, contudo, o conhecimento de níveis gerais desses elementos para o local. A relação sugerida pelos autores está na equação 3 :

$$
E T o=a+b\left(\frac{\Delta}{\Delta+\gamma}\right) \times R s
$$

em que:

ETo - Evapotranspiração de referência, mm.dia- ${ }^{1}$;

$\mathrm{a}--0,3 \mathrm{~mm} \cdot \mathrm{dia}^{-1}$;

$\mathrm{b}$ - fator de ajuste que depende da umidade relativa do ar e da velocidade do período diurno.

Rs - radiação solar à superfície, mm.dia ${ }^{-1}$.

O método de Blaney-Criddle foi desenvolvido relacionando os valores da evapotranspiração mensal com o produto da temperatura média mensal pela percentagem mensal de horas anuais de luz solar, o qual foi modificado pela FAO, incluindo ajustes climáticos locais, sendo assim calculada pela equação 4 (CASTIBLANCO et al., 2016).

$$
E T o=K p\left(0,46 \times T_{a}+8,13\right)
$$

m que:

ETo - Evapotranspiração de referência, mm.dia ${ }^{-1}$;

$\mathrm{k}$ - coeficiente mensal dependente da vegetação, localidade e estação do ano, que varia de 0,5 a 1,2 ;

$\mathrm{p}$ - porcentagem de insolação máxima diária (N) em relação ao horário de insolação teórico do ano (4380 h).

Também utilizado na estimativa da ETo, constitui-se em uma aproximação do método de Penman (FANAYA JÚNIOR et al., 2012). Nesta equação, permanece apenas o saldo de radiação corrigido por um coeficiente empírico “ $\alpha$ ", conhecido como parâmetro de Priestley e Taylor (1972), conforme equação 5.

$$
\text { ETo }=1,26\left[\frac{\Delta}{\Delta+\gamma}\right](R n-G)
$$

em que:

ETo - Evapotranspiração de referência, mm.dia ${ }^{-1}$;

$\gamma$ - constante psicrométrica $\left(\mathrm{kPa}^{\circ} \mathrm{C}^{-1}\right)$;

$\Delta$ - derivada da função de saturação de vapor de água;

$\mathrm{Rn}$ - radiação útil recebida pela cultura de referência $\left(\mathrm{mm} \mathrm{dia}^{-1}\right)$;

$\mathrm{G}$ - fluxo de calor recebido pelo solo ( $\mathrm{mm} \mathrm{dia}{ }^{-}$ $\left.{ }^{1}\right)$.

Para comprar os métodos foram utilizados critérios propostos por Jensen et al.(1990) descritos como Erro-padrão de estimativa (EPE) (6), Erro-padrão de estimativa ajustado pela equação de regressão normal (EPEn) (7) e forçada pela origem (EPEo) (7) - e coeficiente de ajustes das equações lineares completas, como também seus respectivos coeficientes de determinação $\left(\mathrm{R}^{2}\right)$.

$$
E P E=\left(\frac{\Sigma\left(Y_{i}-Y_{m}\right)^{2}}{n-1}\right)^{0,5}
$$

em que: 
$Y_{i}$ - evapotranspiração estimada pelo método $\left(\mathrm{mm} . \mathrm{d}^{-1}\right)$

$\mathrm{Y}_{\mathrm{m}}-$ evapotranspiração estimada pelo método padrão $\left(\mathrm{mm}^{-1} \mathrm{~d}^{-1}\right)$;

$\mathrm{n}$ - número total de observações.

$$
\text { EPEo ou EPEn }=\left(\frac{\Sigma\left(Y_{i c}-Y_{m}\right)}{n-1}\right)^{0,5}
$$

em que:

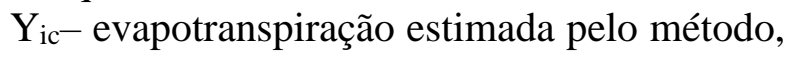
corrigida pelos coeficientes da regressão linear $\left(\mathrm{mm} . \mathrm{d}^{-1}\right)$;

$\mathrm{Y}_{\mathrm{m}}$ - evapotranspiração estimada pelo método padrão $\left(\mathrm{mm}^{\mathrm{d}} \mathrm{d}^{-1}\right)$;

$\mathrm{n}$ - número total de observações.

A análise dos resultados foi baseada em analises de regressão como: precisão (coeficiente de correlação " $r$ ") (8), valores apresentados na Tabela 1; no índice de Willmott "d"(exatidão) por Willmott et al. (1985) (9) e no coeficiente de confiança ou desempenho "c" introduzidos por Camargo e Sentelhas (1997) (10).
Coeficiente de correlação (r):

$$
r=\sqrt{R^{2}}
$$

em que:

$\mathrm{r}$ - coeficiente de correlação; $\mathrm{R}^{2}$ - coeficiente de determinação. Indice de Willmott "d" (exatidão):

$$
d=1-\left[\frac{\Sigma\left(P_{i}-O_{i}\right)^{2}}{\Sigma\left(\left|P_{i}-O\right|+\left|O_{i}-O\right|\right)^{2}}\right]
$$

em que:

$\mathrm{Pi}$ - valor estimado;

Oi - valor observado;

$\mathrm{O}$ - média dos valores observados.

Coeficiente de confiança ou desempenho

(c):

$$
c=r * d
$$

em que:

$\mathrm{r}$ - coeficiente de correlação;

$d$ - índice de Willmott.

Tabela 1. Critérios de interpretação do coeficiente "r" de correlação e índice de desempenho "c".

\begin{tabular}{cccc}
\hline Coeficiente de Correlação (r) & Classificação & Índice de desempenho (c) & Classificação \\
\hline 0,0 a 0,1 & Muito baixa & $>0,85$ & Ótimo \\
0,1 a 0,3 & Baixa & $0,76-0,85$ & Muito Bom \\
0,3 a 0,5 & Moderada & $0,66-0,75$ & Bom \\
0,5 a 0,7 & Alta & $0,61-0,65$ & Mediano \\
0,7 a 0,9 & Muito alta & $0,51-0,60$ & Sofrível \\
0,9 a 1,0 & Quase perfeita & $0,41-0,50$ & Mal \\
& & $\leq 0,40$ & Péssimo \\
\hline
\end{tabular}

\section{RESULTADOS E DISCUSSÃO}

Os dados de temperatura média de máxima, mínima, radiação solar umidade relativa do ar e precipitação podem ser vistos na Figura 2. As maiores temperaturas foram encontradas nos meses de novembro à abril, com valores superiores a $35^{\circ} \mathrm{C}$. O declínio das temperaturas coincidem com início da estação chuvosa que vai de março à setembro, com precipitação média anual de $315 \mathrm{~mm}$. A radiação solar possui similaridade com as curvas de temperatura, possuindo como valores máximos médios os encontrados no período de dezembro e janeiro, atingindo valores acima de 25 Mj.m².dia1. 

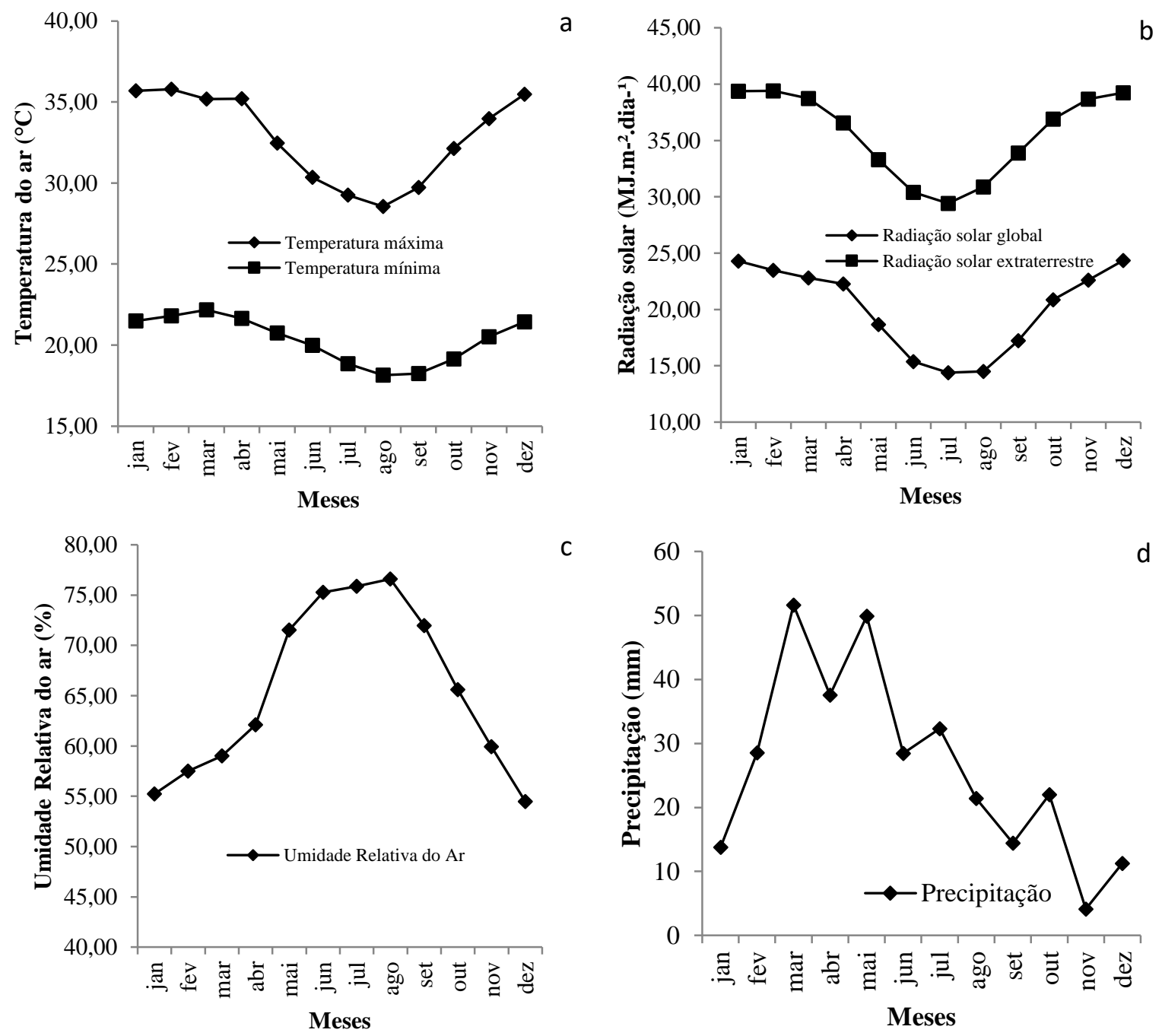

Figura 2. Valores médios mensais para os anos de 2006 a 2013 de temperatura do ar (a), radiação solar (b), umidade relativa (c) e precipitação (d) para o perímetro irrigado Jacaré-Curituba na cidade de Canindé-SE.

A umidade relativa do ar (UR) possui valores baixos ao longo do ano, com máxima de $76,59 \%$, encontrado no mês de julho, e mínima, encontrado no mês de dezembro, com $54,46 \%$, Comparativamente à cidade de Maceió, AL, analisada por Macêdo et al. (2017), possui valores de no mínimo 76\%.

Na Figura 3 está apresentada a análise de regressão linear entre os valores estimados de
ETo, bem como os coeficientes de determinação $\left(\mathrm{R}^{2}\right)$ para regressão completa e forçadas pela origem. Observa-se que os modelos analisados Hargreaves e Samani - HS, Blaney e Criddle - BC e Priestley e Taylor PT (Figura 3b, 3f, 3h) subestimaram o modelo de PM em 27,54, 29,85 e 19,87 \%, respectivamente, enquanto o modelo FAORad (Figura 3d) superestimou em 51,28\%. 

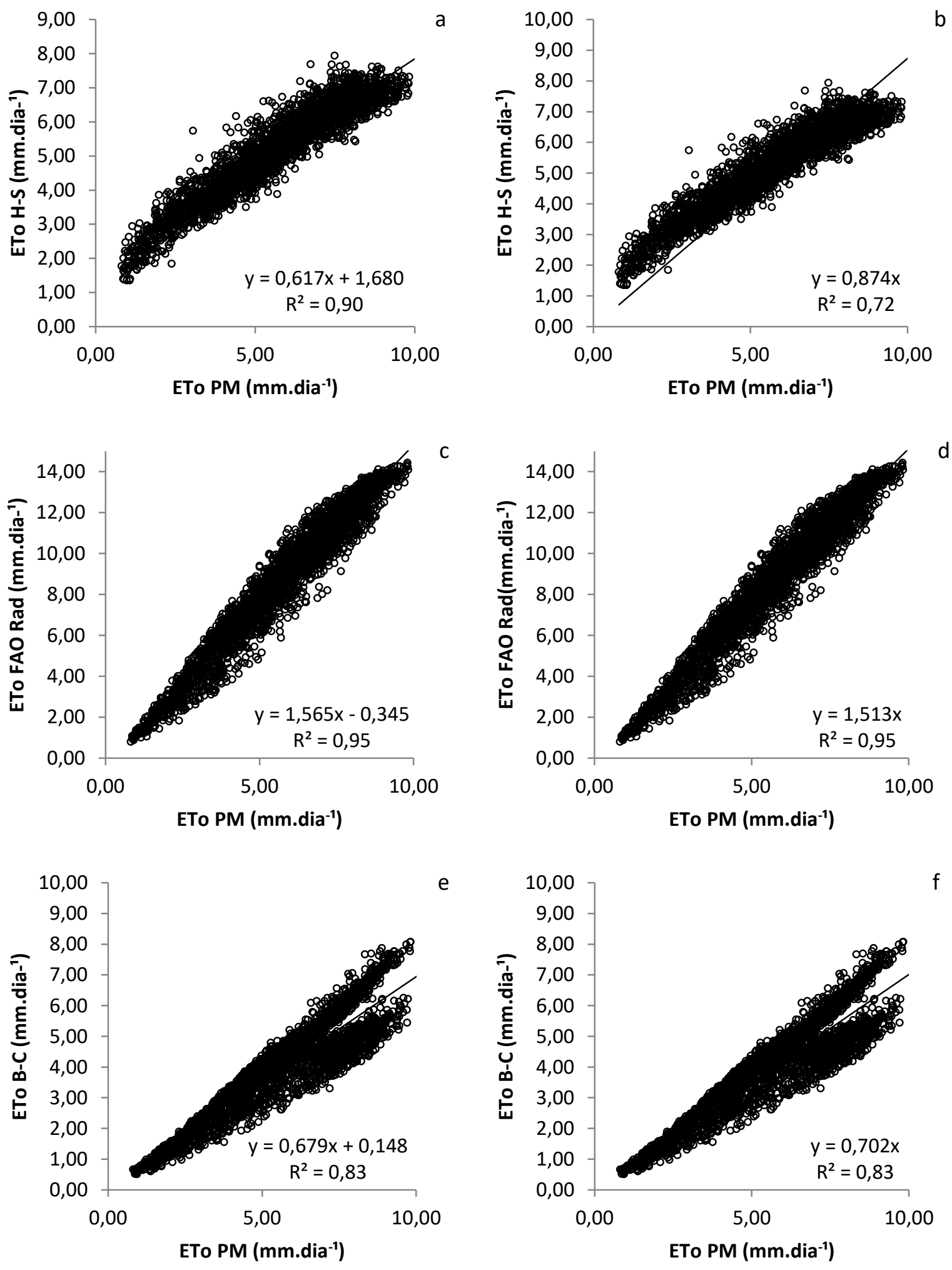

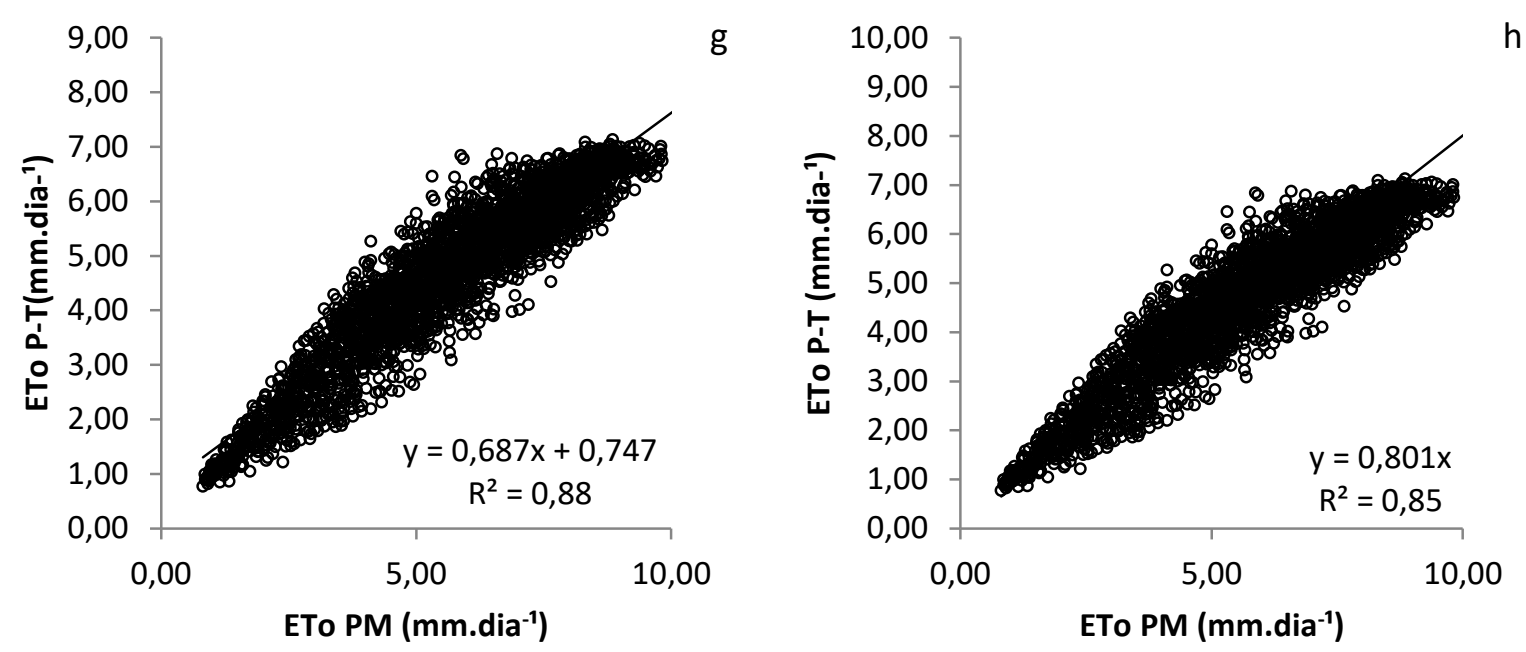

Figura 3. Regressão linear entre valores diários da evapotranspiração de referência (ETo) para o período seco, equação completa e forçada pela origem, estimados segundo o método de Penman-Monteith (PM) (eixo $\mathrm{x}$ ) em relação aos métodos Hargreaves e Samani- HS (a) e (b); FAO-Radiação - FAO-RAd (c) e (d); Blaney e Criddle - BC (e) e (f); Piestley e Taylor - PT (g) e (h), (eixo y).

Segundo Chagas et al. (2013) quando fez uso de métodos empíricos para estimativa de evapotranspiração no município de Rio Real (BA), observou que os métodos FAO-Rad e Hargreaves e Samani superestimaram os valores de ETo em relação ao método de Penman-Monteith em: 9,32 e 20,55\%, respectivamente, apenas Blaney-Criddle subestimou os valores de ETo em 18,14\%.

Gonçalves et al. (2009) observaram uma superestimativa do modelo de HS em relação ao PM em aproximadamente 5\%, para a cidade de Sobral, no Ceará. Borges Júnior et al. (2012), observou uma superestimativa do modelo FAO-Rad e subestimativa do modelo BC para a cidade de Garanhuns-PE.
Observa-se nas Figuras 3a, 3c, 3e, 3g que os modelos apresentaram ótimos ajustes em relação ao modelo padrão, com valores de $\mathrm{R}^{2}$ de aproximadamente $0,90,0,95,0,83$ e $0,88 \%$, respectivamente.

Observa-se na Figura 4 o comportamento da Evapotranspiração de referência ao longo do ano, em meses. Para os meses mais chuvosos, ocorre uma maior aproximação dos valores estimados de ETo em relação ao modelo PM, o modelo HS chega a tingir valores quase idênticos aos de PM. O modelo FAO-Rad possui os dados mais discrepantes. Apesar disso, todos os modelos apresentaram perfil da curva semelhante ao modelo PM.

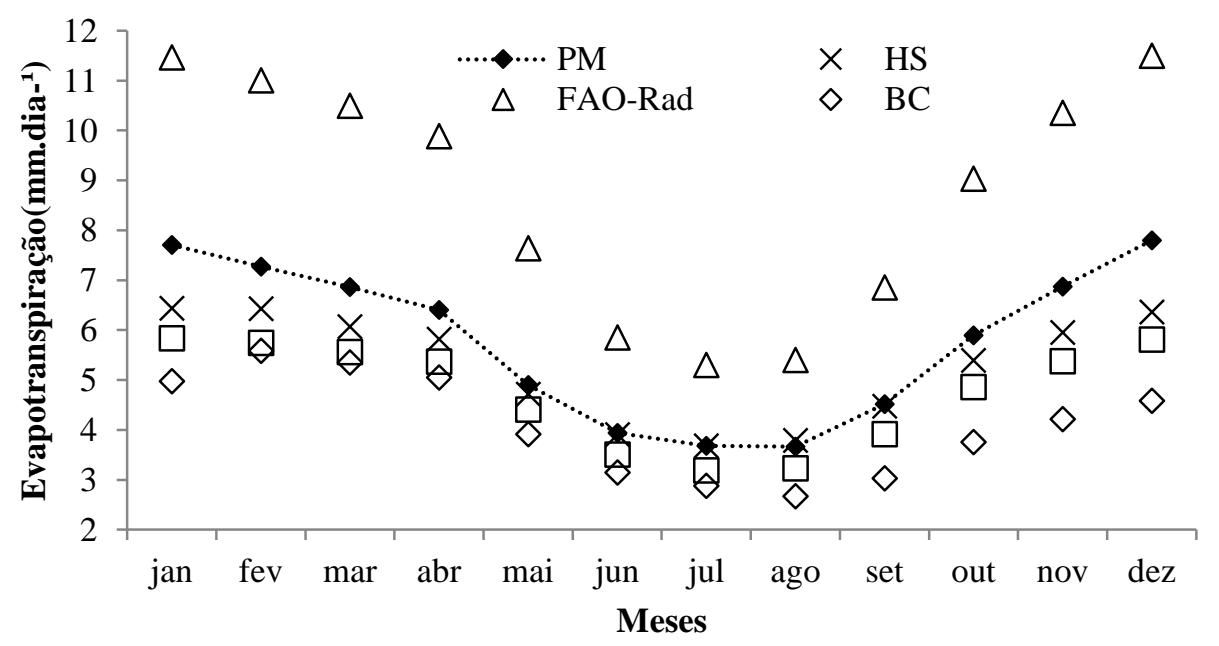


Figura 4. Comportamento da Evapotranspiração de Referência para o perímetro irrigado Jacaré-Curituba pelos modelos de Penman-Monteith (PM), Hargreaves e Samani- HS, FAO-Radiação - FAO-RAd, Blaney e Criddle - BC e Piestley e Taylor - PT.

A Evapotanspiração no perímetro irrigado Jacaré-Curituba segundos os dados do modelo de Penman-Monteith (PM) atinge uma média diária de $5,81 \mathrm{~mm} \cdot \mathrm{d}^{-1}$. A maior média foi para o modelo FAO-Rad, com $8,75{\mathrm{~mm} . \mathrm{d}^{-}}^{-}$ 1 , e menor com o modelo BC, com 4,09 mm.d ${ }^{-}$ 1. O modelo de PT apresentou média de 4,74 mm. $\mathrm{d}^{-1}$ e a média mais próxima foi a de HS com 5,26 mm.d $\mathrm{d}^{-1}$.
De posse dos valores das curvas de regressão normal e forçada pela origem, foram feitos os ajustes dos modelos em relação ao padrão e calculado em seguida os erros de estimativa, quando usados a equação de regressão normal (EPEn) e forçada pela origem (EPEo), observados na Tabela 2, em que também estão os parâmetros estatísticos dos modelos sem os ajustes.

Tabela 2. Tabela com os valores dos parâmetros estatísticos para as correlações entre os métodos de estimativa da ETo em relação ao método padrão de Penman-Monteith, para o perímetro irrigado Jacaré-Curituba, Canindé-SE.

\begin{tabular}{|c|c|c|c|c|c|c|c|c|}
\hline \multicolumn{2}{|c|}{ Correlação } & \multicolumn{7}{|c|}{ Parâmetros estatísticos } \\
\hline $\mathrm{Y}$ & $\mathrm{X}$ & EPE & EPEn & EPFo & $\mathrm{R}^{2}$ & $\mathrm{r}$ & $\mathrm{d}$ & $\mathrm{c}$ \\
\hline HS & PM & 1,05 & 0,65 & 0,80 & 0,90 & 0,95 & 0,91 & 0,86 \\
\hline FAO-Rad & PM & 3,25 & 0,47 & 0,50 & 0,95 & 0,97 & 0,72 & 0,70 \\
\hline BC & PM & 1,95 & 0,84 & 0,89 & 0,83 & 0,91 & 0,75 & 0,69 \\
\hline PT & PM & 1,35 & 0,71 & 0,72 & 0,88 & 0,94 & 0,86 & 0,81 \\
\hline
\end{tabular}

Observa-se que pelos pares de valores $\left(\mathrm{R}^{2} ; \mathrm{r}\right)$ que os métodos de $\mathrm{HS}(0,90 ; 0,95)$, FAO-Rad $(0,95 ; 0,97)$, BC $(0,89$ e 0,83$)$ e PT $(0,88$ e 0,94$)$ tiveram bons ajustes em relação ao modelo padrão - PM, classificando os valores da correlação (r) em "muito alta" para BC e "quase perfeita" para os outros. Chagas et al. (2013) apresentou bons ajustes para os modelos de FAO-RAd e BC, e ajustes inferiores para $\mathrm{HS}$ com valores de aproximadamente $\mathrm{R}^{2}$ e $\mathrm{r}$ de 0,57 e 0,72 , em período seco e 0,77 e 0,88 para períodos úmidos. Gonçalves et al. (2009) apresentaram r de 0,81 para o modelo de HS.

Apesar dos bons ajustes, os erros de estimativa - EPE foram altos, atingindo valores em média superiores a 1,9 mm.dia ${ }^{-1}$. O modelo com menor erro foi o de Hs, e o de maior o FAO-Rad. Após os ajustes observou-se que os melhores resultados, menores erros, foram quando os ajustes foram feitos pela equação de regressão normal. O modelo FAO-Rad apresentou o menor erro, abaixo de 0,5 mm.dia- ${ }^{1}$, demonstrando o potencial do seu uso, se corrigido.

Valores bem menores de EPE foram encontrados por Chagas et al. (2013), Gonçalves et al. (2009) e Borges Júnior et al.(2012), quando utilizaram diferentes métodos de estimativa da ETo.

Com relação ao índice "d" que avalia a exatidão (concordância) dos valores estimados de ETo pelos métodos propostos obtiveram valores entre 0,72 a 0,91 . Os melhores valores são do modelo HS e PT, respectivamente. Os modelo foram classificados, segundo o índice "d" como Bom (FAO-Rad e BC) e ótimo (HS e PT).

O índice "c" foi classificado como Ótimo, para HS; como Muito Bom, para PT e como Bom, para FAO-Rad e BC. Os bons resultados dos modelos, em especial do HS demonstra o potencial que o mesmo tem para seu uso no perímetro irrigado Jacaré-Curituba. 


\section{CONCLUSÕES}

Os métodos analisados em relação ao modelo padrão de Penman-Monteith apresentaram resultados de bom a ótimo;

Quando ajustados pela equação de regressão normal, houve redução dos erros de estimativa em 0,$4 ; 2,78 ; 1,11$ e 0,64 para os modelos HS, FAO-RAD, BC e PT, respectivamente;

Os melhores resultados foram obtidos pelo modelo de Hargreaves e Samani para estimativa da evapotranspiração de referência quando não se dispõe dos valores de evapotranspiração de referência estimados por PM.

\section{REFERÊNCIAS BIBLIOGRÁFICAS}

ALLEN, R. G. et al. Crop evapotranspiration: guidelines fo computing crop water requirements. Irrigation and Drainage Paper 56. p. $300,1998$.

BORGES JÚNIOR, J. C. F., ANJOS, R. J., SILVA, T. J. A., LIMA, J. R. S., ANDRADE,C. L. T. Métodos de estimativa da evapotranspiração de referência diária para a microrregião de Garanhuns, PE. Revista Brasileira de Engenharia Agrícola e Ambiental, v. 16, n. 4, p. 380-390, 2012.

CAMARGO, A. P.; CAMARGO, M. B. P. Uma revisão analítica da evapotranspiração potencial. Bragantia, v. 59, n. 2, p. 125-137, 2000.

CAMARGO, A.P.; SENTELHAS, P.C. Avaliação do desempenho de diferentes métodos de estimativa da evapotranspiração potencial no Estado de São Paulo, Brasil. Rev. Bras. Agrometeorologia, v. 5, n. 1, p. 89-97, 1997.

CASTIBLANCO, C.J.; MENEZES, R. H.; DIAS, A. S. Estimativa da evapotranspiração de referência por diferentes métodos para o município de São Luis - MA. Revista
Brasileira de Agricultura Irrigada, v.10, n.3, p. $621-630,2016$.

CHAGAS, R. M.; FACCIOLI, G. G.; AGUIAR NETTO, A. O.; SOUSA, I. F.; VASCO, A. N.; SILVA, M. G. Comparação entre métodos de estimativa da evapotranspiração de referência (ETo) no município de Rio Real-BA. Irriga, v. 18, n. 1, p. 351-363, 2013.

CODEVASF, Perímetros Irrigados: JacaréCurituba, 2011.

FANAYA JÚNIOR, E. D.; LOPES, A. S. ; OLIVEIRA, G. Q.; JUNG, L. H. Métodos empíricos para estimativa da evapotranspiração de referência para Aquidauana, MS. Irriga, Botucatu, v. 17, n. 4, p. $418-434,2012$

FERNANDES, L. C. Avaliação de diversas equações empíricas de, evapotranspiração. Estudo de caso: Campos dos Goytacazes e Ilha do Fundão-RJ. 2006. 142 f. Dissertação (Mestrado em Engenharia Civil) - COPPE, Universidade Federal do Rio de Janeiro. Rio de Janeiro, RJ, 2006.

GONÇALVES, F. G.; FEITOSA, H. O.; CARVALHO, C. M.; GOMES FILHO, R. R.; VALNIR JÚNIOR, M. Comparação de métodos da estimativa da evapotranspiração de referência para o município de Sobral-CE. Revista Brasileira de Agricultura Irrigada v.3, n.2, p.71-77, 2009.

LIMA, P.E. Evapotranspiração de referência de PenmanMonteith, padrão FAO

(1998), a partir de temperaturas máximas e mínimas de Minas Gerais. 2005. 66p. Tese (Doutorado em Meteorologia Agrícola) Universidade Federal de Viçosa, 2005.

MACÊDO, K. A.; ARRAES, F. D. D. ; LIMA JUNIOR, J. C. ; OLIVEIRA, W.C. ,; ARAUJO, Y. R.; Ajuste dos parâmetros do modelo de Hargreaves E Samani para 
estimativa da evapotranspiração de referência em escala diária para Maceió-AL. Revista Brasileira de Agricultura Irrigada, v.11, n.3, p. 1484 - 1491, 2017.

MENDONÇA, J. C.; SOUSA, E. F.; BERNARDO, S.; DIAS, G. P.; GRIPPA, S. Comparison of estimation methods of reference crop evapotranspiration (ETo) for Northeren Region of Rio de Janeiro State, Brazil., Revista Brasileira de Engenharia
Agrícola e Ambiental v. 7, n. 2, p. 275-279, 2003.

RAVAZZANI, G., CORBARI, C., MORELLA, S., GIANOLI, P., \& MANCINI, M. Modified HargreavesSamani equation for the assessment of reference evapotranspiration in Alpine river basins. Journal of Irrigation and Drainage Engineering, v. 138, n. 7, p. 592599, 2011. 